\title{
Formation and mitigation of halo particles in the Spallation Neutron Source linac
}

\author{
D. Jeon, ${ }^{1}$ J. Stovall, ${ }^{1,4}$ A. Aleksandrov,${ }^{1}$ J. Wei, ${ }^{2}$ J. Staples,${ }^{3}$ R. Keller,${ }^{3}$ L. Young, ${ }^{4}$ H. Takeda, ${ }^{4}$ and S. Nath ${ }^{4}$ \\ ${ }^{1}$ Oak Ridge National Laboratory, Oak Ridge, Tennessee 37830 \\ ${ }^{2}$ Brookhaven National Laboratory, Upton, New York 11973 \\ ${ }^{3}$ Lawrence Berkeley National Laboratory, Berkeley, California 94720 \\ ${ }^{4}$ Los Alamos National Laboratory, Los Alamos, New Mexico 87545
}

(Received 10 April 2002; published 11 September 2002)

\begin{abstract}
A halo generation mechanism in the nonperiodic lattices such as the SNS (Spallation Neutron Source) linac MEBT [medium-energy beam transport between radio-frequency quadrupole and DTL (drift tube linac)] is reported. We find that the nonlinear space charge force resulting from large transverse beam eccentricity $\sim 2: 1$ in the $\sim 1.6$ m-long MEBT chopper section is responsible for halo formation. As a result, the beam distribution, based on the front end emittance measurements and multiparticle simulation studies, develops halo that leads to beam loss and radioactivation of the SNS linac. Designing lattices with transverse beam eccentricity close to 1:1 suppresses this kind of halo generation. Modifying the MEBT optics and introducing adjustable collimators in the MEBT significantly reduced beam losses in the coupled cavity linac, which is a preferred scheme for mitigating halo. It turns out that the DTL collimation does not effectively remove halo and presents a risk of overheating drift tubes.
\end{abstract}

DOI: $10.1103 /$ PhysRevSTAB.5.094201

PACS numbers: 29.27.Bd, 41.75.Cn

\section{INTRODUCTION}

The SNS (Spallation Neutron Source) accelerator system is designed to accelerate intense proton beams to energy of $1 \mathrm{GeV}$, delivering more than $1.4 \mathrm{MW}$ (upgradable to $2 \mathrm{MW}$ ) of beam power to the neutron production target [1]. The peak current in the linac is $38 \mathrm{~mA}$ and the macropulse average current is $26 \mathrm{~mA}$. The SNS linac has the following structure: ion source, LEBT (low-energy beam transport), RFQ (radio-frequency quadrupole), MEBT (medium-energy beam transport), DTL (drift tube linac), CCL (coupled cavity linac), and SCL (superconducting linac). A primary concern is potential damage and radioactivation of accelerator components resulting from uncontrolled beam losses. A major source of loss is beam halo that intercepts the bore of the linac.

Beam dynamics simulations of the SNS linac show that the beam halo develops at low energy, but some halo particles survive acceleration to higher energies before being lost primarily on the CCL bore. This particle loss at higher energies results in radioactivation of the CCL. In order to find ways to mitigate this halo related beam loss, we conducted studies to identify the sources and mechanism of halo formation. It turns out that the MEBT is the largest contributor to FE (front end) halo generation in the SNS linac.

It should be noted that the MEBT consists of nonperiodic lattices to facilitate chopping for the ring injection. Halo generation mechanisms in such nonperiodic lattices are less known. On the other hand, mechanisms in periodic lattices are relatively well understood. One of such is a mismatch, which has long been known as a mechanism of halo generation [2,3]. And Jeon et al. [4] first showed explicitly that space charge induced resonance causes a significant emittance growth and halo formation in high intensity rings such as the SNS accumulator ring. Space charge induced resonance effects in high intensity linacs have also been extensively studied [5].

This paper identifies a halo generation mechanism in nonperiodic lattices such as the SNS linac MEBT. Understanding of this halo generation mechanism is important to most other spallation neutron sources being designed such as the ESS or the JHF projects because they have a MEBT-like section to do beam chopping for ring injection. In Sec. II, discussions on the halo generation mechanism in nonperiodic lattices are presented. Lowenergy halo mitigation/collimation schemes for the SNS linac are presented in Sec. III. Feasibility of DTL collimation is discussed in Sec. IV, followed by the conclusion in Sec. V.

Figure 1 shows the measured beam emittance in the LEBT upon which we based our beam-dynamics simulations. The upper row of plots shows $x-x^{\prime}$ projections and the second row shows $y-y^{\prime}$. The two colored plots in the first column show the raw emittance data. The second column shows the "analyzed" data after applying a threshold to eliminate noise and spurious signals. The third column shows the macroparticle distribution that we derived from the analyzed data and that we used in our numerical simulations. The derivation algorithm assumed a particular type of distribution for the unmeasured correlations in the real beam distribution. The bottom row shows these four projections generated for the numerical distribution: $x-y$, $x^{\prime}-y^{\prime}, x-y^{\prime}$, and $x^{\prime}-y$. For more detailed information on the FE, please refer to [6]. 


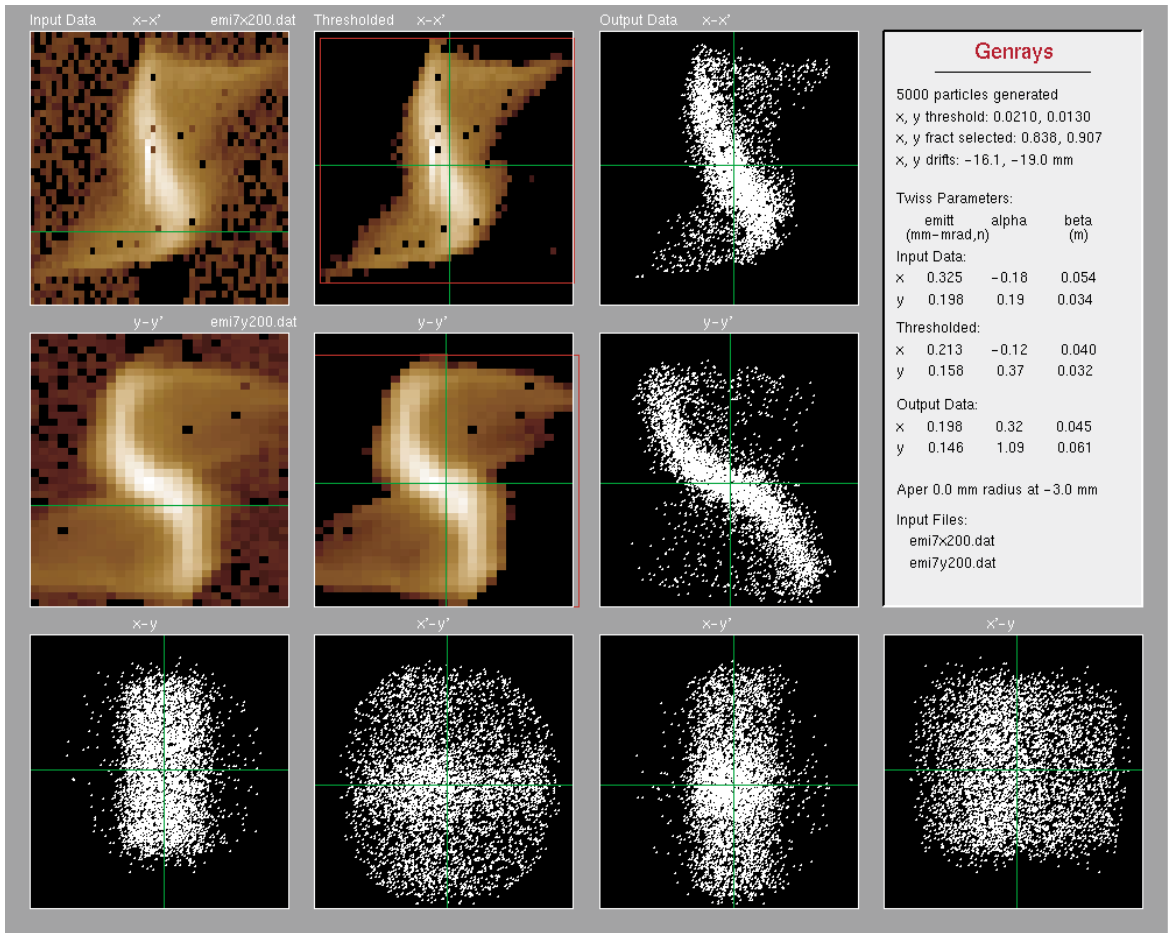

FIG. 1. (Color) Phase-space projections of the measured and simulated LEBT beam. Leftmost colored plots are raw data of LEBT emittance measurements. The colored plots in the second column are filtered data. And rightmost plots are generated beam distribution based on the filtered data.

\section{NONLINEAR SPACE CHARGE FORCE INDUCED HALO IN MEBT}

The MEBT is the largest contributor to beam halo before DTL. This is clearly displayed by comparing the MEBT input beam and MEBT output beam. Figure 2 shows the
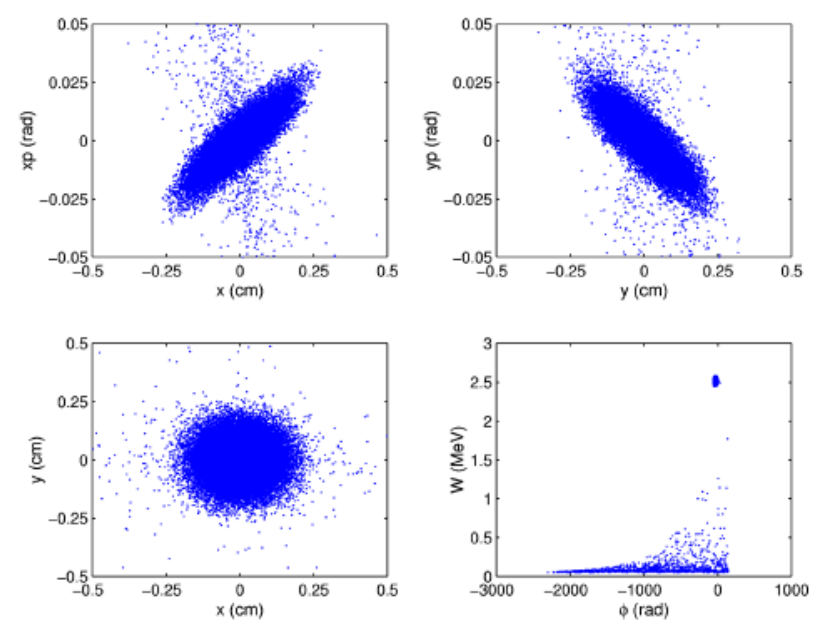

FIG. 2. (Color) Transverse and longitudinal phase-space projections of the beam at the MEBT entrance. The scattered particles are low-energy particles, lost immediately in the MEBT. The number of macroparticles is 87000 . This beam is used throughout the study. The normalized $x(y)$ rms emittance is $0.36(0.28)$ $\pi$ mm mrad. transverse phase-space projections of the measured beam tracked to the MEBT entrance (after the RFQ). The particles that appear to be scattered in the angular dimension correspond to low-energy particles $(\sim 0.1 \mathrm{MeV})$ that are destined to be lost longitudinally immediately and are of no concern. Otherwise, this beam is only slightly worse than one originating from an initial water-bag distribution at the RFQ entrance. There is no sign of beam distortion in phase space at the entrance to MEBT.

However, Fig. 3 shows the well-developed horizontal halo in transverse phase-space projections of the beam at the end of the MEBT (before the DTL), which is a result of the MEBT optics. In the DTL, these tails develop into a halo that is inseparable from the core of the beam, making halo collimation in DTL inefficient as discussed in Sec. IV.

To better understand MEBT optics, the horizontal (middle curve) and vertical (bottom curve) envelope profiles of the $\operatorname{sqrt}(5) *$ rms beam size in the MEBT are plotted in Fig. 4. The top curve is the longitudinal envelope profile. The beam is squeezed vertically to clear the vertical deflection plates of both the chopper and antichopper and relaxed horizontally. This arrangement is necessary to have $90^{\circ}$ zero-current betatron phase advance between the chopper box and the chopper target in the middle (between the chopper target and the antichopper box as well). However, this $1.6 \mathrm{~m}$-long chopper section with a large beam eccentricity is the source of halo formation shown in the upper left plot of Fig. 3. 

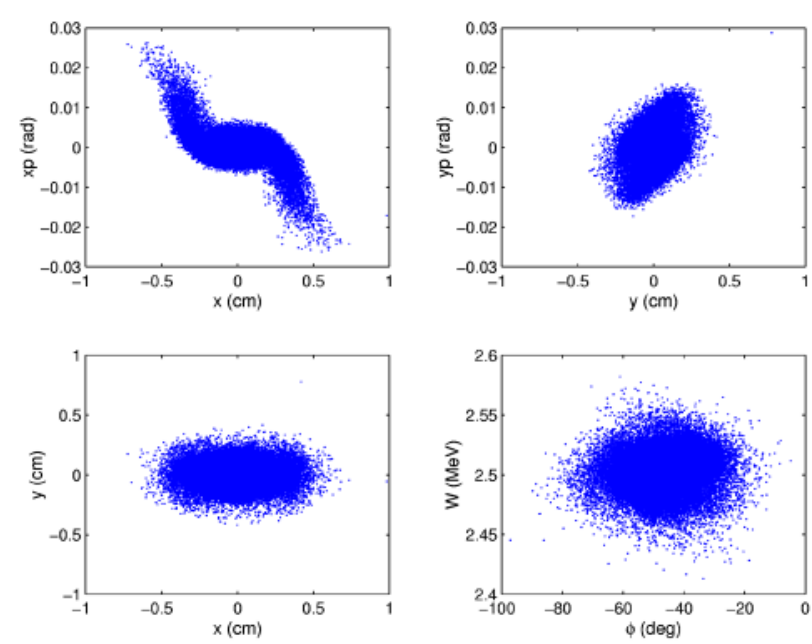

FIG. 3. (Color) Transverse and longitudinal phase-space projections of the beam at the end of MEBT. On the contrary to the MEBT input beam, well-developed horizontal halo is observed. This is a result of the MEBT optics. The normalized $x(y) \mathrm{rms}$ emittance is $0.21(0.20) \pi \mathrm{mm}$ mrad.

Figure 5 shows the electric field (in arbitrary units) on top of real space projections of beam distribution at the chopper target (in the middle of the MEBT) where the beam eccentricity is $\sim 2: 1$. The beam is wide in $x$ and narrow in $y$. The $E x$ becomes nonlinear beyond $x=0.5 \mathrm{~cm}$, which is well inside the core. This means that the outer part of the core with $|x|>0.5 \mathrm{~cm}$ (marked as the "potential halo") is subject to nonlinear space charge force and their phase advance is quite different from the inner part of the core seeing linear space charge force. The phase advance difference over the $1.6 \mathrm{~m}$ chopper section leads to severe beam distortions in horizontal phase space. In the case of $E y$, only a small fraction of halo particles sees nonlinear space charge force. This is why the tail develops mainly in $x$ phase space by the end of the MEBT.

To suppress halo formation induced by large transverse beam eccentricity, the optics of lattices should make the beam as round as possible. For the purpose of studying, we modified the entire MEBT optics to reduce beam eccentricity as shown in Fig. 6 (compare with Fig. 4). Reducing the beam eccentricity indeed suppresses the halo formation as shown in Fig. 7 that depicts the beam profiles at $171 \mathrm{MeV}$ before and after optics modification. However,
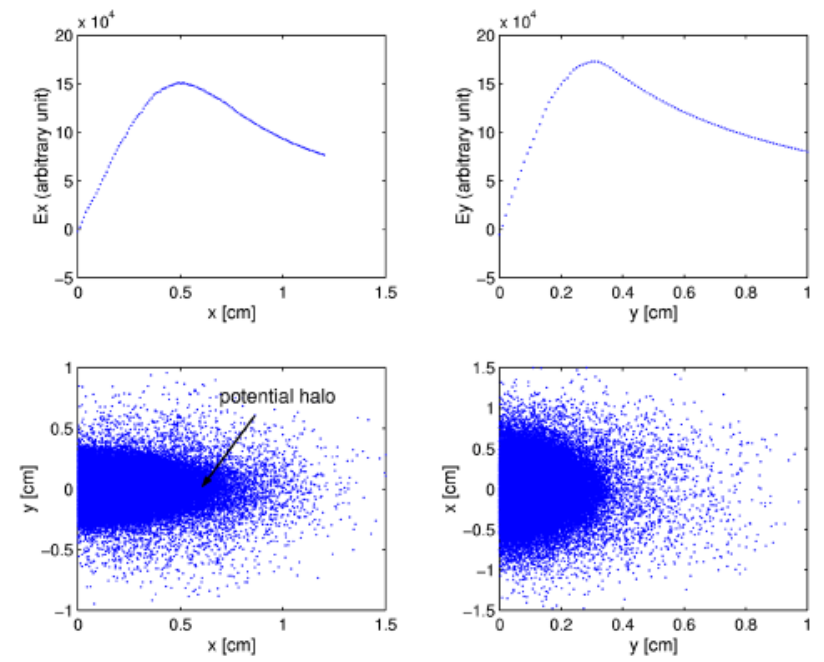

FIG. 5. (Color) Plots of $E$ field and real space projections of beam distribution. Ex is plotted along the $x$ axis and $E y$ along the $y$ axis. The unit of $E$ field is arbitrary. $x$ rms beam size is $3.40 \mathrm{~mm}$ and $y \mathrm{rms}$ beam size is $1.71 \mathrm{~mm}$.

modification of the entire MEBT optics is not viable to facilitate the beam chopping for ring injection. At least the first half of the MEBT should not be modified, while the second half can be modified. The proposed MEBT optics modification is discussed in Sec. III B.

In the DTL, the energy associated with the horizontal tails quickly gets redistributed, resulting in a halo in both horizontal and vertical emittance projections. We are, of course, concerned with any increase in the effective emittance of the beam, but more importantly in any increase in the real-spatial size of the beam, which increases the risk of interception with the linac bore. The top radial particle distribution in Fig. 7 shows that the halo extends beyond the $1.5 \mathrm{~cm}$ physical bore radius at the end of the CCL even without any machine imperfections. Throughout the CCL the focusing strength of the transverse lattice gradually weakens to smoothly match the focusing strength in the SCL. As a result, the beam size is largest near the end of the CCL, so that most of the beam loss occurs at energies near $171 \mathrm{MeV}$. By including machine imperfections in the simulations we readily see the locations of "hot spots" caused by beam loss, as shown in Fig. 8. Ten linac runs are made with machine imperfections. The maximum, minimum, and average beam losses are plotted. Most hot

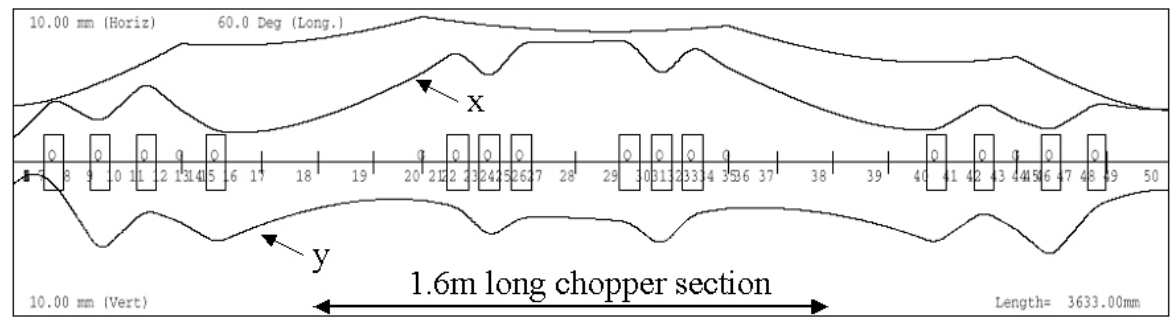

FIG. 4. Trace 3D beam envelope profiles in the MEBT before modification. 


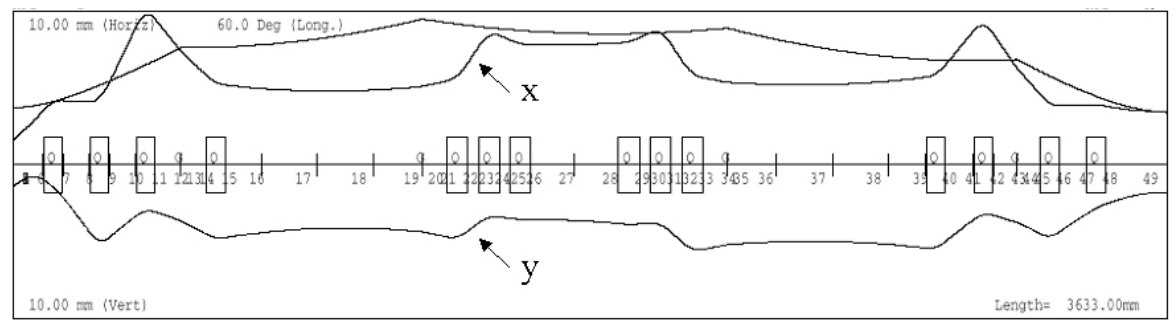

FIG. 6. Trace 3D beam envelope profiles of modified MEBT to reduce the beam eccentricity.

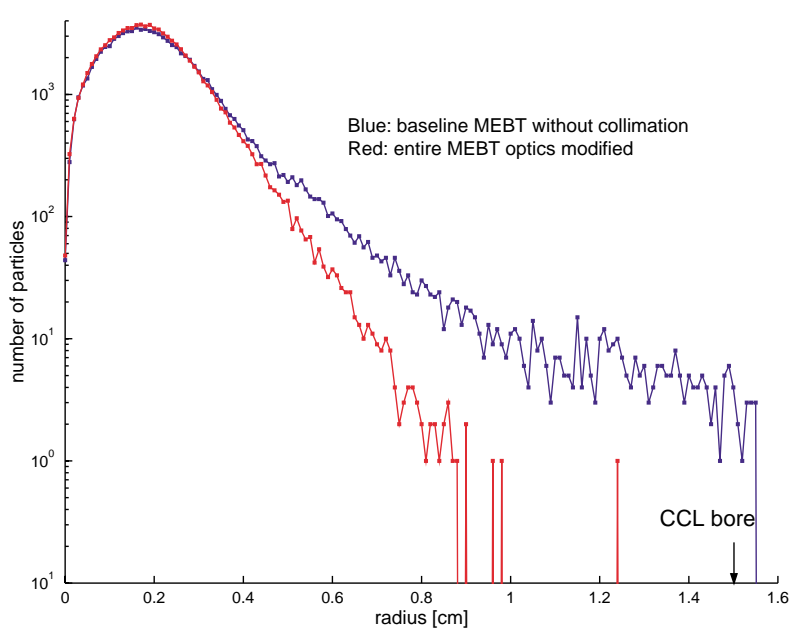

FIG. 7. (Color) Radial particle distributions at CCL (171 MeV point) before (top curve) and after reducing the beam eccentricity (bottom curve). After modification, halo is reduced significantly.

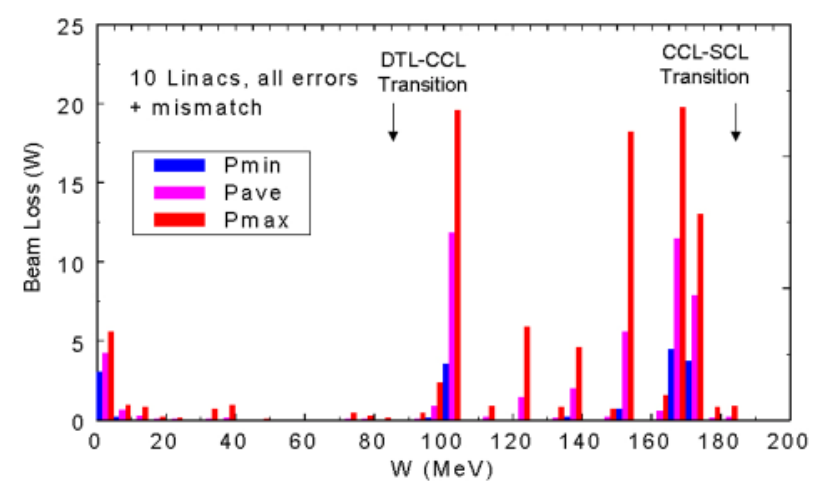

FIG. 8. (Color) Beam loss plots with machine imperfections and mismatch for the original MEBT. Most beam loss takes place in the CCL and at transitions between structures.

spots are in the CCL and there is a minor hot spot at the MEBT-DTL interface. In general, very little beam loss takes place in the DTL.

Our simulations indicate that, without cleaning, the beam halo results in beam loss primarily in the CCL. Furthermore, the halo effectively increases beam emittance and causes more particles to miss the injection foil. We have investigated the effectiveness and feasibility of four schemes for reducing the halo, including the DTL collimation. Given the uncertainty of the beam distribution from the LEBT, our goal is to implement a robust system that can accommodate varying commissioning and operating beam conditions.

\section{SNS LINAC LOW ENERGY COLLIMATION PLANS}

We propose a scheme for halo mitigation that reduces the opportunity for formation of transverse tails and trims the tails before they have an opportunity to mix with the beam core. The proposed scheme utilizes adjustable collimators at the location of the MEBT chopper target and/or adjustable collimators in the antichopper box (in the case that the antichopper is unnecessary).

\section{A. MEBT scraping}

There are only a few places where collimators will fit in the MEBT. One convenient place is at the chopper target. Figure 9 shows the layout of the MEBT with the chopper target and antichopper box indicated by arrows. A pair of adjustable horizontal collimators would be installed in the chopper target box (at the red arrow). The chopper target itself is located above the midplane to intercept the beam that is deflected upward. Collimators mounted on horizontal actuators will not interfere with the function of the target. This assembly is shown schematically in Fig. 10. This collimator implementation has the advantage that it is readily adjustable to accommodate the actual beam conditions, which are expected to vary with different operating conditions such as beam current, ion-source performance, LEBT, RFQ, and MEBT tuning. The other advantage is that the proposed collimators can be cooled easily. The adjustable collimators are designed to scrape up to about $20 \%$ of beam power when they are made of carbon/carbon composite [7], in other words, up to about $10 \%$ by each of the two adjustable horizontal collimators.

To study the effectiveness of using a MEBT collimator with the current MEBT optics shown in Fig. 4, we fixed the horizontal MEBT collimator aperture at $\pm 8 \mathrm{~mm}$, scraping off $2 \%$ of the beam. Now there is no beam loss in an imperfection-free DTL/CCL. Figure 11 shows that, without machine imperfections, $84 \%$ of the beam tail with $r>9 \mathrm{~mm}$ is removed. This result suggests that MEBT collimation at the proposed location is effective. 


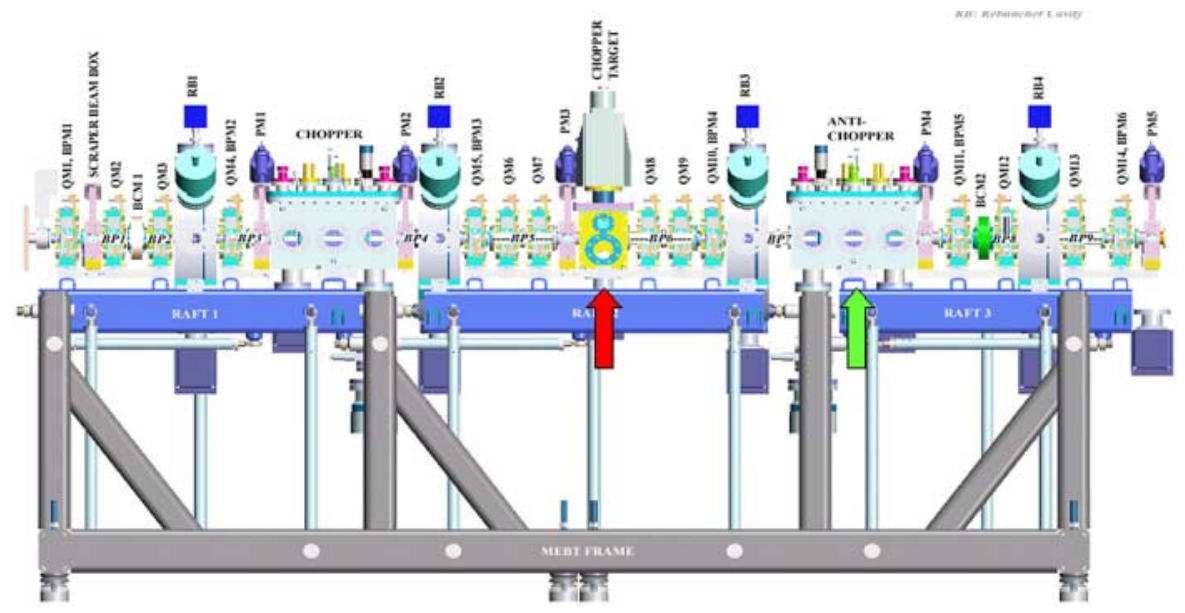

FIG. 9. (Color) Schematic layout of the MEBT indicating the location of adjustable horizontal collimators at the chopper target (red arrow). A second 4-jaw adjustable collimator could replace the antichopper as a backup (green arrow).

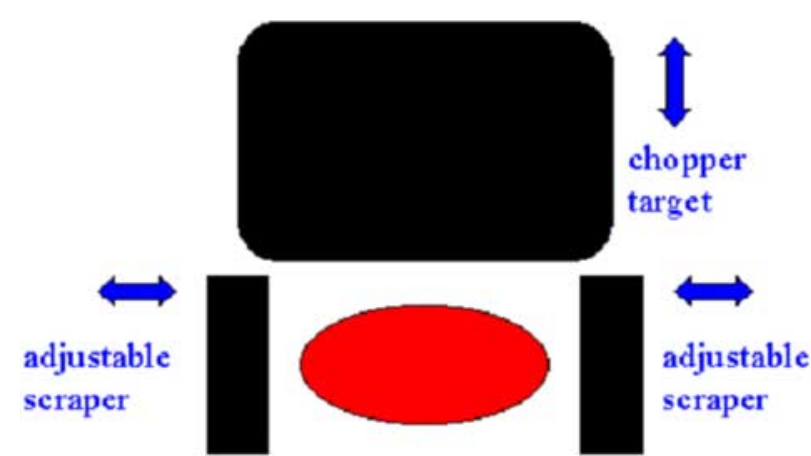

FIG. 10. (Color) Schematic drawing of adjustable MEBT collimators and chopper target.

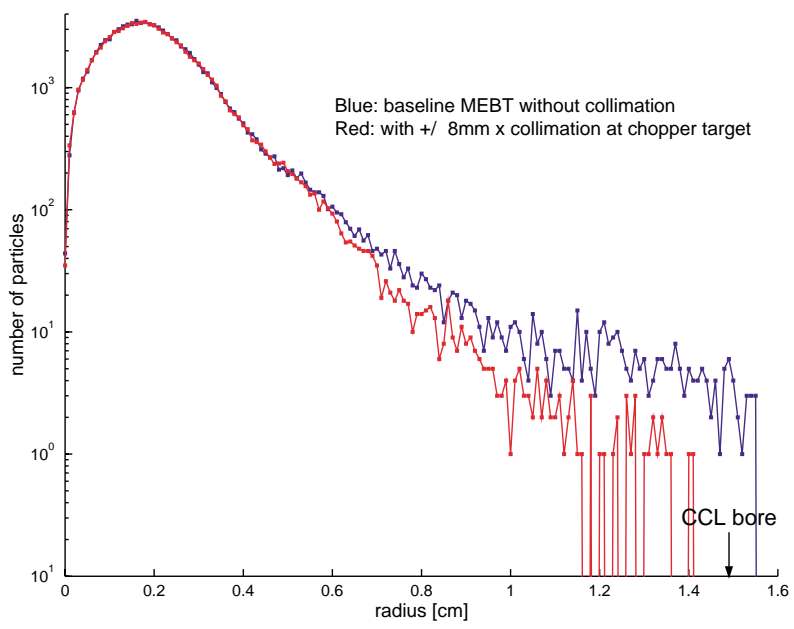

FIG. 11. (Color) Beam profile at $171 \mathrm{MeV}$ with (upper curve) and without MEBT collimation (lower curve) at $\pm 8 \mathrm{~mm}$. Halo is reduced by $84 \%$. This means that $84 \%$ of the beam with $r>9 \mathrm{~mm}$ is removed. CCL bore radius is $1.5 \mathrm{~cm}$.

\section{B. Alternative MEBT optics}

The primary cause of halo generation is nonlinear spacecharge forces acting on a beam having a large transverse eccentricity as discussed in Sec. II. This condition occurs in both the MEBT chopper and the antichopper where the beam is compressed vertically to facilitate chopping. The MEBT antichopper ideally operates at a vertical betatron phase advance of $90^{\circ}$ (zero-current phase advances) from the chopper target, and $180^{\circ}$ from the MEBT chopper, to restore any unchopped portion of the beam to its "original" position in phase space. Figure 4 shows that this condition produces a beam profile that is symmetric about the chopper target.

In an alternative design, we preserve the $90^{\circ}$ phase advance from the chopper to the target, but we relax it to $63^{\circ}$ from the target to the antichopper to reduce the transverse beam eccentricity. The resulting beam cross section is more circular as shown in Fig. 12. Now, the antichopper no longer restores a partially chopped portion of the beam to its original (on-axis) position in phase space, if indeed that were desirable. Also, the beam now has a larger vertical extent and approaches the antichopper plates as designed. Alternative modes of antichopper operation are under study.

This simple modification to the optics alone reduces the formation of transverse tails substantially and improves the beam quality in the downstream linac. Figure 13 shows that $87 \%$ of the beam tails with $r>9 \mathrm{~mm}$ at $171 \mathrm{MeV}$ is removed. The halo reduction is comparable to the effect of MEBT collimation with the baseline MEBT optics.

\section{Hybrid halo reduction solution}

We also investigated the effectiveness of adding MEBT collimation in combination with the alternative optics design. In this scenario, we added MEBT collimation at 


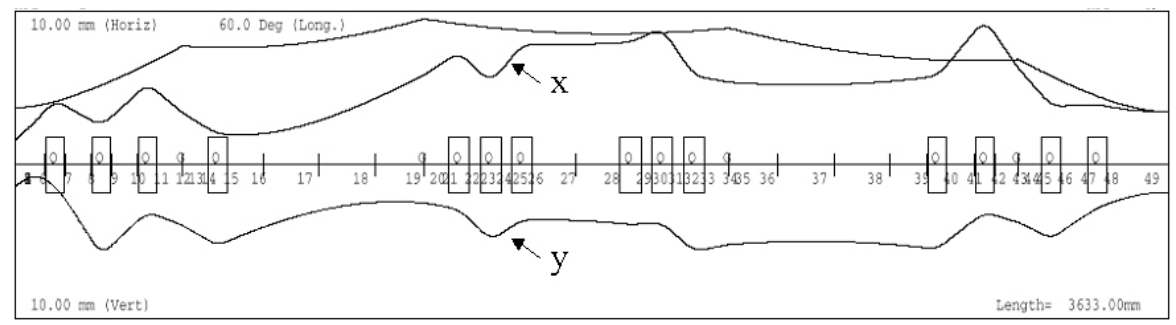

FIG. 12. Trace 3D beam envelope profiles of the modified MEBT optics yielding a more circular cross section in the antichopper.

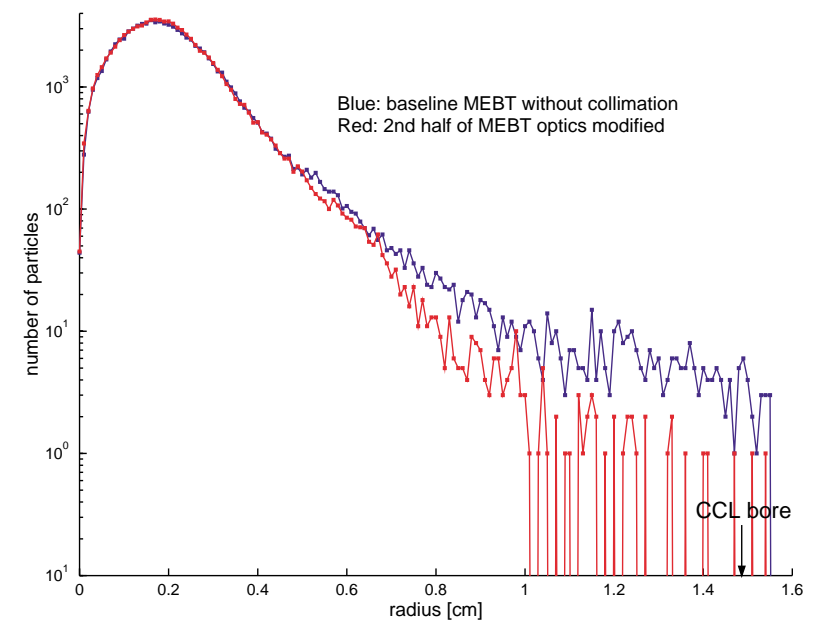

FIG. 13. (Color) Radial beam distribution at $171 \mathrm{MeV}$ before (upper curve) and after (lower curve) MEBT optics modification. $87 \%$ of the beam tail with $r>9 \mathrm{~mm}$ is removed just due to optics change.

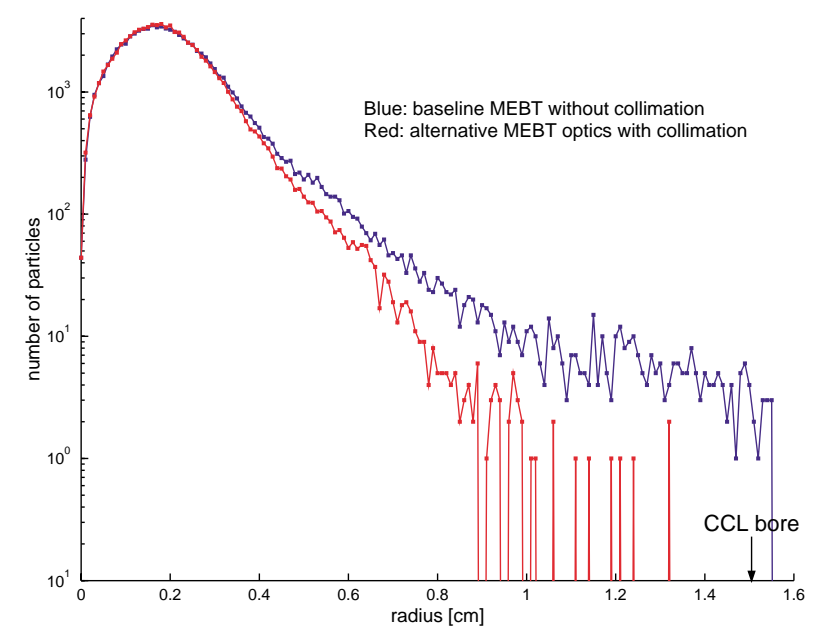

FIG. 14. (Color) Radial beam distribution at $171 \mathrm{MeV}$ for the hybrid halo mitigation solution. $97 \%$ of the halo with $r>$ $9 \mathrm{~mm}$ is removed with the combination of two collimators and modified MEBT optics (lower curve). The upper curve corresponds to the baseline performance. the two locations indicated by arrows in Fig. 9. We adjusted horizontal collimators to $\pm 8 \mathrm{~mm}$ at the chopper target and introduced a square collimator of $\pm 6 \mathrm{~mm}$ in the antichopper box. The $\pm 8 \mathrm{~mm}$ horizontal collimators are collimating $2.2 \%$ of the beam and the square collimators of $\pm 6 \mathrm{~mm}$ at the antichopper box are scraping $1.0 \%$ of the beam. Figure 14 shows the radial beam distribution at $171 \mathrm{MeV}$ resulting from this hybrid solution. Compared with the baseline case $97 \%$ of the halo with $r>9 \mathrm{~mm}$ is removed. We also studied the effectiveness of the proposed scheme for the increased peak current of $54 \mathrm{~mA}$ rather than $38 \mathrm{~mA}$. There is enough safety margin even for this case.

Modifying the MEBT to create a more circular beam profile clearly eliminates much of the halo seen in the baseline design. In addition, with the introduction of variable collimators, we expect to reduce uncontrolled beam loss associated with halo to a manageable level. And the $2 \mathrm{MW}$ upgrade path does not impact this scheme, because it is to be accomplished by increasing the energy gain in the superconducting linac.

\section{DTL COLLIMATION}

We also explored the possibility of DTL collimation of the SNS linac. The original MEBT is used without optics modification in the tracking studies in order to single out the effectiveness of the beam collimation in the DTL. The focusing lattice in the DTL is FFODDO, where O means empty drift tubes. We considered inserting circular collimators in the first 11 empty drift tubes. The bore radius of drift tubes is $12.5 \mathrm{~mm}$. By using only empty drift tubes, we avoid the possibility of overheating and possibly approaching the Curie point of the permanentmagnet quadrupole lenses (PMQs). In addition, the beam is nearly round in the empty drift tubes making collimation by circular collimators more effective.

The first five lattice periods, spanning 30 drift tubes, represent a depressed phase advance of about $2 \pi$ rad. Therefore, placing collimators in the first 10 or 11 empty drift tubes should trim off the halo, assuming that the halo remains on the perimeter of the beam. The collimator size would have to follow the beam size. Figure 15 shows 


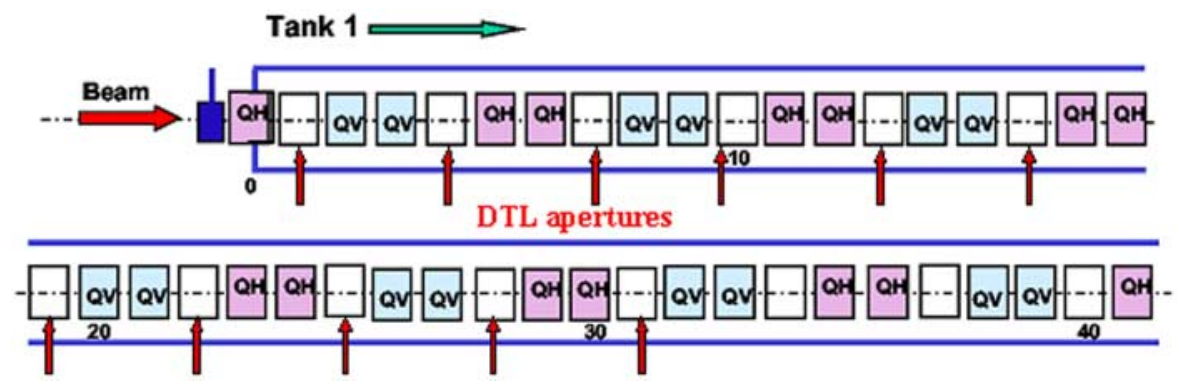

FIG. 15. (Color) Schematic diagram of DTL tank 1 showing the location of proposed collimators.

schematically the first half of DTL tank 1 . The colored drift tubes contain PMQs. The vertical red arrows show the proposed locations of collimators.

We predict the effectiveness of the collimation scheme by identifying the halo particles "at risk" as the outermost $1 \%$ of the upper curve in Fig. 7, which corresponds to those having a radius greater than $9 \mathrm{~mm}$. We then track these particles through the DTL. Figure 16 shows the real space projections of the beam at the chopper target and at the first seven locations of the drift tube collimators. The at risk particles are plotted in red.

Halo particles have more transverse energy than do core particles. They oscillate through the core with large amplitudes, spending only part of their time on or near the beam perimeter. The projections in Fig. 16 clearly show halo particles inside the core of the beam. In fact, the halo is completely unobserved at some points (e.g., drift tubes 1 and 16). The tails created in the MEBT have become so well integrated with the core of the beam that drift tube collimation is not effective.

To evaluate the effectiveness of DTL collimators, we looked at the radial particle distribution at $171 \mathrm{MeV}$ for several collimator radii. To evaluate their feasibility, we calculated the amount of energy deposited in each drift tube from the scraped beam. This energy represents an addition to the rf thermal load on each drift tube. The water-cooling circuit must remove this heat in order to hold the structure on resonance.

We first considered $8 \mathrm{~mm}$-radius circular collimators (drift tube bore radius is $12.5 \mathrm{~mm}$ ) and transported the beam without including machine imperfections such as misaligned drift tubes. The collimators reduce the bore cross section by $36 \%$, but trim just $0.22 \%$ from the beam. The left-hand plot of Fig. 17 is the expected power deposited in the collimating drift tubes. The maximum power of $\sim 5 \mathrm{~W}$ deposited in drift tube 10 adds $10 \%$ to the rf heat load. The expected beam-loss profile compared with the beam sizes in Fig. 16 shows that the beam completely clears the collimators in drift tubes 13, 16, and 19 , so there is no beam power added to these drift tubes.

The first dipole corrector in DTL tank 1 is in drift tube 49 , so there is no steering spanning the proposed collimators. Besides, there is no beam-position monitor (BPM) in DTL tank 1. With this lack of steering, it is prudent to study the effects of machine imperfections. The right-hand plot of Fig. 17 summarizes the simulated particle dynamics in 100 linacs that included random alignment errors. The plot shows the maximum and minimum power lost at
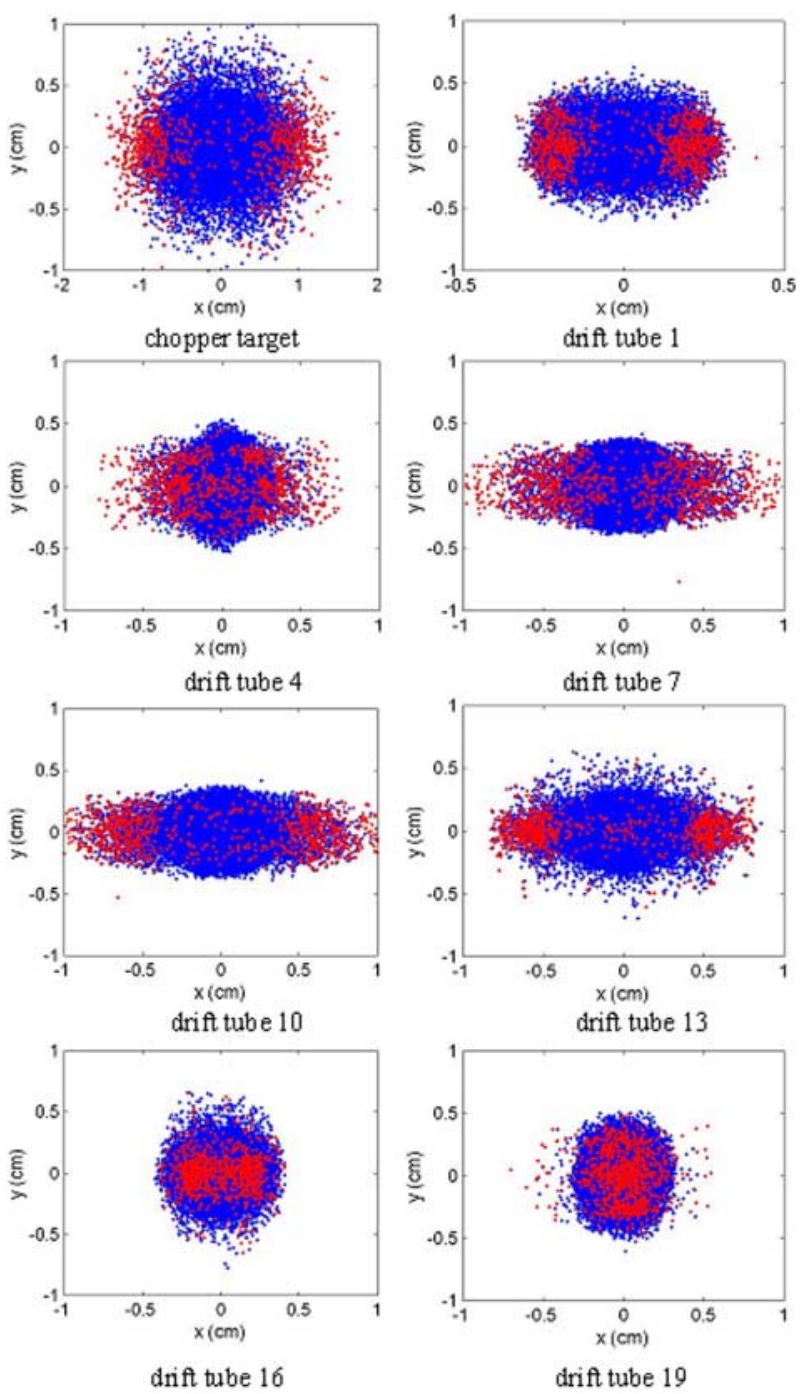

FIG. 16. (Color) Real space projections of the beam distribution at the MEBT chopper target and at the first seven of the proposed DT collimators. Halo particles "at risk" are plotted in red. 

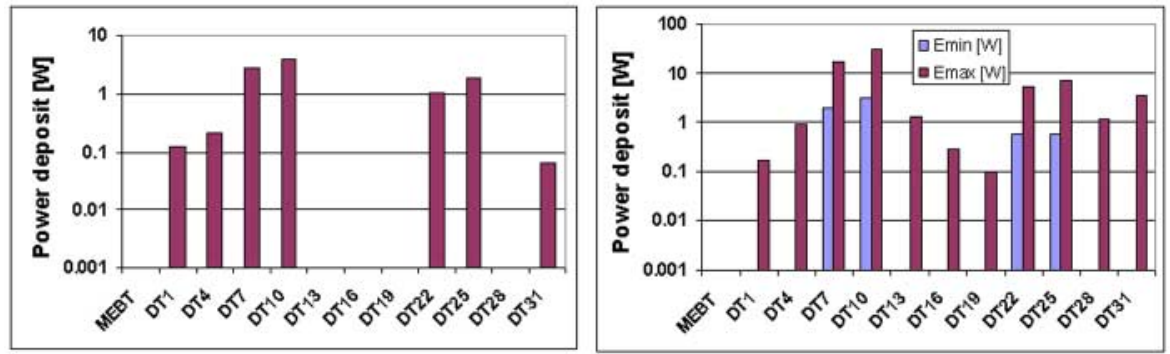

FIG. 17. (Color) Expected power deposited in $8 \mathrm{~mm}$ radius DTL collimators. The left-hand plot assumes no errors. The right-hand plot summarizes the results of 100 linac runs with errors. The bore radius of drift tubes is $12.5 \mathrm{~mm}$.

each circular collimator. The maximum power, again at drift tube 10 , was $31 \mathrm{~W}$, adding $65 \%$ to rf heating.

The radial particle distributions plotted in Fig. 18 show the effectiveness in reducing halo by $8 \mathrm{~mm}$ radius collimators. The blue curve is the radial beam distribution at $171 \mathrm{MeV}$ without collimation, and the red curve is the distribution after being trimmed. Even though $0.22 \%$ of the beam has been trimmed from the beam there is no observable reduction in the beam halo.

Next we considered $6 \mathrm{~mm}$-radius collimators (drift tube bore radius is $12.5 \mathrm{~mm}$ ), which reduces the bore cross section by $64 \%$. Indeed, the beam halo at $171 \mathrm{MeV}$ is reduced as shown in Fig. 19. The smaller-aperture collimators remove over $1 \%$ of the beam and reduce the current in the halo $(r>9 \mathrm{~mm})$ by $90 \%$. Unfortunately, the energy deposited in the $6 \mathrm{~mm}$ apertures is excessive.

The left-hand plot of Fig. 20 shows that, excluding machine imperfections, the beam power deposited in drift tube 10 would double the design thermal load. Including machine imperfections (right-hand plot of Fig. 20), the maximum expected power deposited in drift tube 22 is $444 \mathrm{~W}$, which is $\sim 6$ times the design cooling capacity of this drift tube.

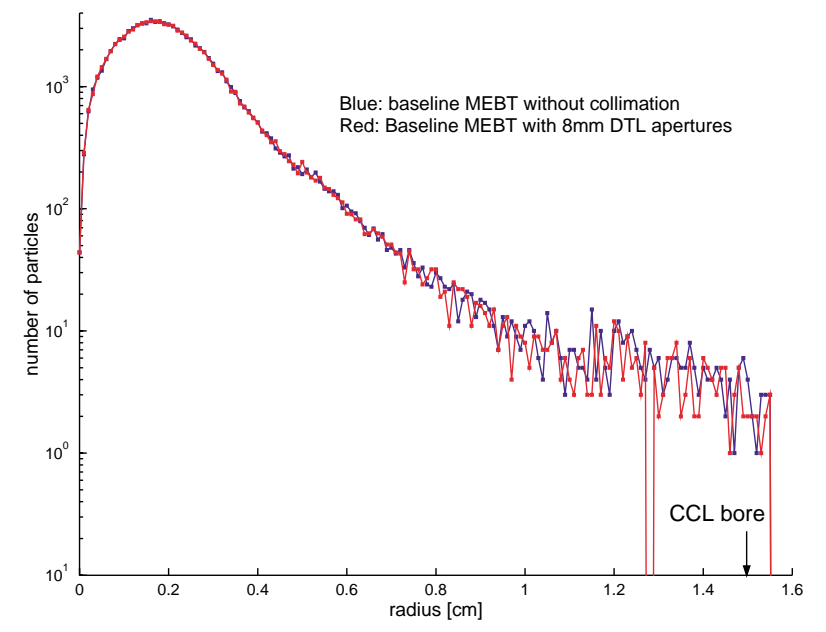

FIG. 18. (Color) Radial beam distribution at $171 \mathrm{MeV}$ with and without DTL collimation. DTL collimators of $8 \mathrm{~mm}$ radius do not reduce beam halo.
The random misalignment of the PMQs steers the beam, so the beam centroid is seldom on axis. Without BPMs in the low-energy end of tank 1 we have no way to steer the MEBT beam onto the DTL axis. As a consequence, the beam will scrape the bore asymmetrically as shown in Fig. 21. Although we did not quantify this effect, it is clear that even small-aperture drift tube collimators would not assure a significant reduction in the halo, but would guarantee serious heating problems.

We conclude that placing collimators in drift tubes is neither an effective nor safe approach to removing halo particles that are expected to be lost in an uncontrolled way.

(i) Collimators with too small an aperture $(\sim 6 \mathrm{~mm}$ radius) are required to effect any useful reduction in the beam halo.

(ii) Severe thermal loading of the drift tubes will exceed the ability of the present drift tube cooling design.

(iii) Collimators of fixed size and shape lack the flexibility required to accommodate beam matching and steering.

(iv) Asymmetric scraping is expected to result from unpredictable construction and steering errors that would nullify any expected benefit.

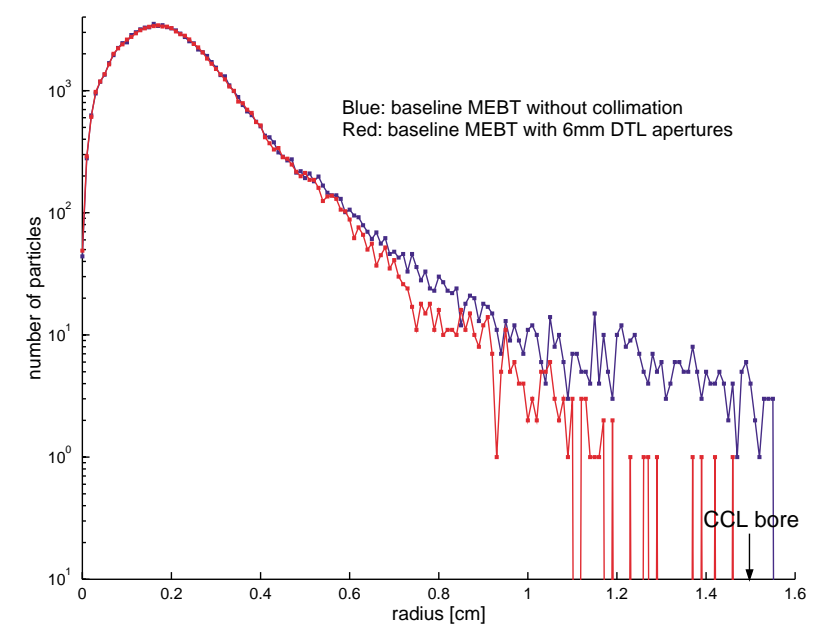

FIG. 19. (Color) Radial beam distribution at $171 \mathrm{MeV}$ with and without DTL collimation. Apertures of $6 \mathrm{~mm}$ radius reduce beam halo by $90 \%$, but result in excessive drift tube heating. 

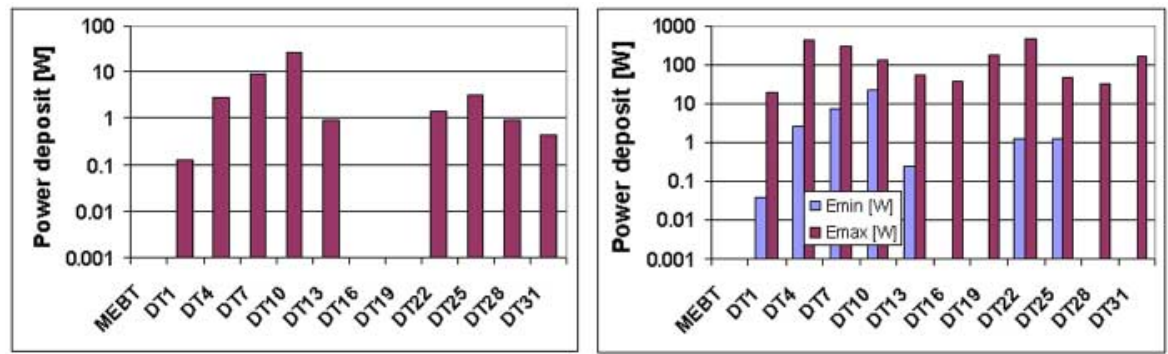

FIG. 20. (Color) Expected power deposited in $6 \mathrm{~mm}$ radius DTL collimators. The left-hand plot assumes no errors. The right-hand plot summarizes the results of 100 linac runs with errors. The drift tube bore radius is $12.5 \mathrm{~mm}$.

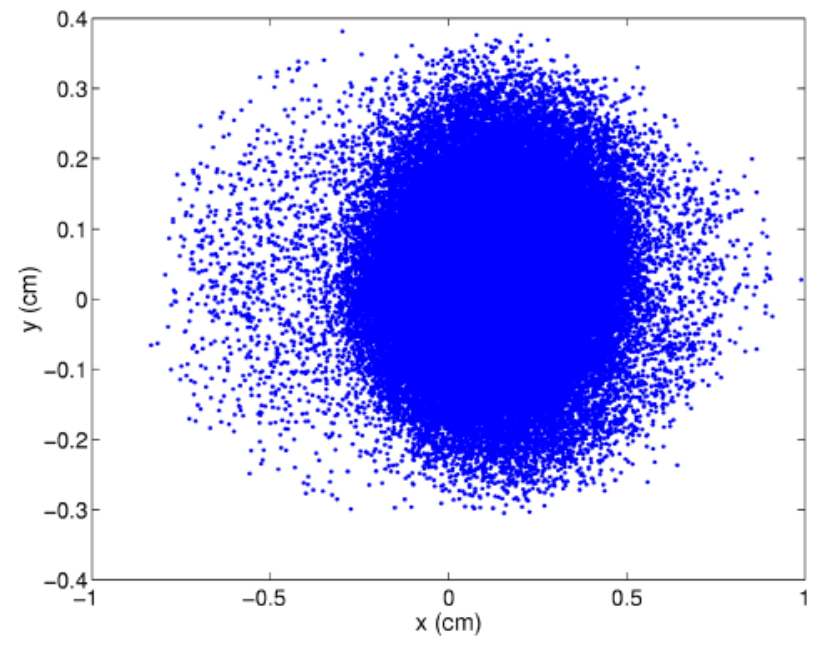

FIG. 21. (Color) Real space beam distribution at the end of DTL tank 1 for one set of random machine imperfections. The resulting asymmetric scraping leaves a significant halo.

\section{CONCLUSION}

The nonlinear space charge force resulting from large transverse beam eccentricity is responsible for the halo formation in the FE of the SNS linac. Modifying the MEBT optics and introducing adjustable collimators at the chopper target as needed is a preferred scheme for mitigating halo by preempting its formation. The hybrid solution does not involve any redesign. Because the quadrupole lenses and collimators in the MEBT are all adjustable, this scheme is adaptable to any operational scenario. We also studied the effectiveness of halo collimation in other places of the linac. After investigating the potential for mitigating halo by introducing collimators in the DTL, we conclude that this scheme does not remove halo effectively. Collimators with too small an aperture are required to reduce halo significantly and results in severe thermal loading of the drift tubes. Asymmetric scraping, resulting from machine imperfections, would nullify any potential benefit. Fixed apertures would limit our ability to accommodate any variety of beam conditions that will arise during the commissioning and normal operation.

\section{ACKNOWLEDGEMENT}

This work is supported by the SNS Project managed by UT-Battelle, LLC, under Contract No. DE-AC0500OR22725 for the U.S. Department of Energy. SNS is a partnership of six national laboratories: Argonne, Brookhaven, Jefferson, Lawrence Berkeley, Los Alamos, and Oak Ridge. We would like to thank J. Billen (LANL) for his edition and support.

[1] J. Wei et al., in Proceedings of the 2001 Particle Accelerator Conference, Chicago, IL (IEEE, Piscataway, NJ, 2001), p. 319.

[2] P. M. Lapostolle, IEEE Trans. Nucl. Sci. 18, 1101 (1971); F. J. Sacherer, ibid. 18, 1105 (1971); J. D. Lawson, P. M. Lapostolle, and R. L. Gluckstern, Part. Accel. 5, 61 (1973); E. P. Lee and R. K. Cooper, ibid. 7, 83 (1976).

[3] S. Y. Lee and A. Riabko, Phys. Rev. E 51, 1609 (1995); A. Riabko et al., Phys. Rev. E 51, 3529 (1995).

[4] D. Jeon et al., Phys. Rev. E 60, 7479 (1999); D. Jeon, in Proceedings of the 2001 Particle Accelerator Conference, Chicago, IL (Ref. [1]), p. 3191.

[5] I. Hofmann and O. Boine-Frankenheim, Phys. Rev. Lett. 87, 034802 (2001); I. Hofmann et al., Phys. Rev. Lett. 86, 2313 (2001).

[6] R. Keller et al., in Proceedings of the 2001 Particle Accelerator Conference, Chicago, IL (Ref. [1]), p. 70.

[7] Private communication with S. Kim: carbon/carbon developed for the fusion reactor is considered. See, for example, http://www.toyotanso.com. 